\title{
Sequential Blind Source Extraction For Quasi-Periodic Signals With Time-Varying Period
}

\author{
Thato Tsalaile*, Member, IEEE, Reza Sameni, Student Member, IEEE, Saeid Sanei, Senior Member, IEEE, \\ Christian Jutten, Fellow, IEEE, and Jonathon Chambers, Senior Member, IEEE
}

\begin{abstract}
A novel second-order-statistics-based sequential blind extraction algorithm for blind extraction of quasi-periodic signals, with time-varying period, is introduced in this paper. Source extraction is performed by sequentially converging to a solution that effectively diagonalizes autocorrelation matrices at lags corresponding to the time-varying period, which thereby explicitly exploits a key statistical nonstationary characteristic of the desired source. The algorithm is shown to have fast convergence and yields significant improvement in signal-to-interference ratio as compared to when the algorithm assumes a fixed period. The algorithm is further evaluated on the problem of separation of a heart sound signal from real-world lung sound recordings. Separation results confirm the utility of the introduced approach, and listening tests are employed to further corroborate the results.
\end{abstract}

Index Terms-Blind source extraction (BSE), quasi-periodic, second-order statistics, statistical nonstationarity, time-varying period.

\section{INTRODUCTION}

B LIND SOURCE extraction (BSE) has received much research attention because of its potential utility in a wide range of applications including many in biomedical signal processing. The problem arises when linear, instantaneous mixtures or observations, generated as a set of signals are mixed by traversing an unknown medium, essentially without delay, need to be processed to estimate or recover a number or all of the original sources. One of the important and challenging issues in BSE is how to extract specific sources of interest. This requires the proper use of prior information about the sources or the mixing operation in forcing the algorithm to extract the sources of interest rather than any arbitrary sources. The objective of blind source separation (BSS), on the other hand, is to simultaneously recover or estimate all the original sources from their mixtures. Compared with BSS, BSE provides more flexibility and has

Manuscript received January 14, 2008; revised May 7, 2008. First published July 15, 2008; current version published April 15, 2009. Asterisk indicates corresponding author.

${ }^{*} \mathrm{~T}$. Tsalaile is with Loughborough University, Loughborough LE11 3TU, U.K. (e-mail: t.k.tsalaile@lboro.ac.uk).

R. Sameni is with the Department of Electrical Engineering, Sharif University of Technology, Tehran 11365-9517, Iran, and also with the Institut National Polytechnique de Grenoble (INPG), Grenoble 38031, France.

S. Sanei is with the Center of Digital Signal Processing, Cardiff University, Cardiff 44000, U.K.

C. Jutten is with the Department Images-Signal (DIS), Grenoble Images, Speech, Signal and Control Laboratory (GIPSA, 300 people), Grenoble 38031, France.

J. Chambers is with the Department of Electronic and Electrical Engineering, Loughborough University, Loughborough LE11 3TU, U.K.

Color versions of one or more of the figures in this paper are available online at http://ieeexplore.ieee.org.

Digital Object Identifier 10.1109/TBME.2008.2002141 some potential advantages over BSS, in terms of computational complexity and extraction of only the sources of interest.

Over the last decade or so, several approaches have been developed for the solution of both BSS and BSE problems, which are based on either second-order or higher order statistics of the data. Typically, the higher order techniques consist of two steps: a whitening step for exploiting the second-order statistics and a rotation step for exploiting the higher order statistics. They require few assumptions apart from the statistical independence of the sources, and therefore, have generally been the preferred approach to the solution of BSE and BSS. Higher-order-statisticsbased solutions include [1]-[4]. Second-order statistics methods, on the other hand, have the advantage of requiring shorter data records due to their reduced sensitivity to small sample estimation errors, and do not limit the number of Gaussian sources that can be separated to one (see, for instance, [5]-[8]). As opposed to higher-order methods, second-order methods operate in a semiblind context, since their derivation usually requires that certain additional assumptions are made on the nature of the original signals, such as statistical nonstationarity of the sources, presence of temporal structure in stationary signals, or cyclostationarity [5]-[8]. Such information is usually available in certain biomedical applications, for instance, in physiological signals such as the ECG, and should be exploited.

Several algebraic-block-based methods exist that exploit the temporal correlations of the source signals, and perhaps the best known is the second-order blind identification (SOBI) algorithm [9]. Consistent with the operation of batch algorithms, the original SOBI algorithm entails prewhitening the data, followed by the (approximate) joint diagonalization of a set of covariance matrices at different time lags, thus potentially allowing separation of sources based on their temporal structure. However, in the SOBI algorithm, the time lags at which the covariance matrices are jointly diagonalized are fixed and are not matched to the extraction of a quasi-periodic signal with timevarying period. Furthermore, computational complexity of this algorithm is generally substantially greater than sequential algorithms due to the need to diagonalize a number of sample covariance matrices, and therefore, will not be considered further in this paper. Related algorithms that are essentially based on a similar principle can be found in [10] and [11].

Recently, a sequential algorithm was developed for a class of periodic signals in [12]. In that work, however, the signals, although periodic, have a constant or fixed period. In this paper, we exploit the combination of the sequential blind source extraction (BSE) algorithm using second-order statistics based on the approximate joint diagonalization (AJD) of autocorrelation 
matrices [13] and the time-varying lag (period) calculation procedure recently proposed in [20], and thus, introduce a novel sequential blind source extraction algorithm for the extraction of quasi-periodic signals with time-varying period. This paper is motivated by the observation that the majority of physiological signal measurements (for example, ECG) exhibit some degree of periodicity and statistical nonstationarity. The nonstationarity manifests itself as variations in period as a function of time. This makes the assumption of a fixed period (as in [12]) invalid for the ECG signal, and perhaps many other biomedical signals. To the best of our knowledge, a sequential BSE algorithm that is matched to such variations in the signal period has not been discussed previously. Moreover, using a time-varying period can help with the extraction of a specific desired source.

To this end, a time-varying period $\tau_{t}$, which is estimated for each new cycle-to-cycle interval of the quasi-periodic source to be extracted, is incorporated in the sequential blind extraction algorithm. Source extraction is performed by sequentially converging to a solution that effectively diagonalizes the autocorrelation matrices at lags $\tau_{t}$ corresponding to the different periods.

The rest of the paper is organized as follows. Problem formulation, in the context of BSE using second-order statistics is presented in Section II. In Section III, we present and incorporate the concept of time-varying period in the problem formulated in Section II. We present the simulation results in Section IV. In Section V, we present results of applying the new algorithm to extraction of a heart sound signal (HSS) from real-world lung sound recordings. A summary and concluding remarks are given in Section VI.

\section{PROBLEM Formulation}

We consider the real-valued signal generating model

$$
\mathbf{x}(t)=\mathbf{A} \mathbf{s}(t)+\mathbf{n}(t)
$$

where $\mathbf{s}(t)=\left[s_{1}(t), s_{2}(t), \ldots, s_{N}(t)\right]^{T}$ is a column vector of $N$ mutually uncorrelated zero-mean unknown source signals, $\mathbf{A}=\left[\mathbf{a}_{1}, \mathbf{a}_{2}, \ldots, \mathbf{a}_{N}\right]$ is an $N \times N$ invertible unknown mixing matrix, $\mathbf{x}(t)=\left[x_{1}(t), x_{2}(t), \ldots, x_{N}(t)\right]^{T}$ is a column vector of $N$ observed sensor signals, $\mathbf{n}(t)=\left[n_{1}(t), n_{2}(t), \ldots, n_{N}(t)\right]^{T}$ denotes a column vector of additive white Gaussian zero-mean measurement noise, $\mathbf{a}_{i}$ is the $i$ th column of $\mathbf{A}$, and $[\cdot]^{T}$ and $t$, respectively, denote the vector transpose and the discrete time index. In the discussion that follows, we proceed with the noiseless model of (1) by dropping the noise term $\mathbf{n}(t)$, but we show the effect of the noise on the algorithm in the simulation section (Section IV).

Based on the assumption that the sources are spatially uncorrelated and wide sense stationary, the time-lagged autocorrelation matrix $\mathbf{R}_{k}$ can be defined as

$$
\mathbf{R}_{k}=E\left[\mathbf{x}(t) \mathbf{x}^{T}\left(t-\tau_{k}\right)\right], \quad k=1,2,3, \ldots, K
$$

where $K$ is the index of the maximum time lag, i.e., $\tau_{K}$ and $E[\cdot]$ denotes the statistical expectation operator.

The vector $\mathbf{x}(t)$ in (1) (ignoring the noise term) is a linear combination of the columns of matrix $\mathbf{A}$, i.e., $\mathbf{a}_{i}$ s. Therefore, the most intuitive way to extract the $i$ th source is to project $\mathbf{x}(t)$ onto the space in $\mathbb{R}^{N}$ orthogonal to, denoted by $\perp$, all of the columns of $\mathbf{A}$ except $\mathbf{a}_{i}$, i.e., $\left\{\mathbf{a}_{1}, \ldots, \mathbf{a}_{i-1}, \mathbf{a}_{i+1}, \ldots, \mathbf{a}_{N}\right\}$. Henceforth, by defining a vector $\mathbf{q} \perp\left\{\mathbf{a}_{1}, \ldots, \mathbf{a}_{i-1}, \mathbf{a}_{i+1}, \ldots, \mathbf{a}_{N}\right\}$ and setting $\mathbf{t} \equiv \mathbf{a}_{i}$, together with adopting oblique projector notation [14], gives

$$
y(t) \mathbf{t}=\mathbf{E}_{\mathbf{t} \mid \mathbf{q}^{\perp}} \mathbf{x}(t)
$$

where $y(t)$ is an estimate of one source, $\mathbf{q}^{\perp}$ is a subspace in $\mathbb{R}^{N}$ orthogonal to $\mathbf{q}$, i.e., the space spanned by $\left\{\mathbf{a}_{1}, \ldots, \mathbf{a}_{i-1}, \mathbf{a}_{i+1}, \ldots, \mathbf{a}_{N}\right\}$, and $\mathbf{E}_{\mathbf{t} \mid \mathbf{q}^{\perp}}=\left(\mathbf{t q}^{T}\right) /\left(\mathbf{q}^{T} \mathbf{t}\right)$ is the oblique projection of $\mathbf{t}$ onto the space $\mathbf{q}^{\perp}$. By omitting the scalar $1 /\left(\mathbf{q}^{T} \mathbf{t}\right)$ and dropping $\mathbf{t}$ from both sides of (3) results in

$$
y(t)=\mathbf{q}^{T} \mathbf{x}(t) .
$$

In BSE based on second-order statistics, both vectors $\mathbf{t}$ and $\mathbf{q}$ are unknown. In order to extract one source, we adopt the same approach and assumptions as in [13, Sec. III], i.e., the following cost function is exploited to find these vectors

$$
[\hat{\mathbf{t}}, \hat{\mathbf{q}}, \hat{\mathbf{d}}]=\arg \min _{\mathbf{t}, \mathbf{q}, \mathbf{d}} J(\mathbf{t}, \mathbf{q}, \mathbf{d})
$$

where $J(\mathbf{t}, \mathbf{q}, \mathbf{d})=\sum_{k=1}^{K}\left\|\mathbf{R}_{k} \mathbf{q}-d_{k} \mathbf{t}\right\|^{2}, \mathbf{d}=\left[d_{1}, d_{2}, \ldots, d_{K}\right]^{T}$ is the column vector of unknown scalars, and $\|\cdot\|$ denotes the Euclidean norm. The cost function in (5) utilizes the fact that for BSE, $\mathbf{R}_{k} \mathbf{q}$ should be collinear with $\mathbf{t}$, incorporating the coefficients $d_{k}$ that provide $\mathbf{t}$ with proper scaling. The trivial answer for (5) is its immediate global minimum point when $\mathbf{t}=\mathbf{q}=\mathbf{d}=\mathbf{0}$. This solution has been avoided by imposing the condition $\|\mathbf{t}\|=\|\mathbf{d}\|=1$. Minimization of the cost function (5) with respect to $\hat{\mathbf{q}}$ leads to the identification of vector $\mathbf{q}$ in (4) that can therefore be used to extract one of the sources. It is, however, worth noting that the actual extracting vector is given by $\mathbf{q} /\left(\mathbf{q}^{T} \mathbf{t}\right)$ due to earlier omission of the scaling factor $1 /\left(\mathbf{q}^{T} \mathbf{t}\right)$ in order to arrive at (4). The convergence of (5) is rather difficult to prove analytically in the time domain due to the product term $d_{k} \mathbf{t}$ in (5). The formal analytical proof of the convergence is a subject of future research.

\section{A. Signal Extraction Algorithm}

By employing the sequential approximate digitalization algorithm (SDA) proposed in [13], the cost function (5) is minimized by adjusting its parameters alternatively as follows.

Stage 1: Freeze both $\mathbf{t}$ and $\mathbf{d}$ and adjust $\mathbf{q}$. Taking the gradient of $J$ with respect to q leads to analytical solution for $\mathbf{q}$ as $\partial J / \partial \mathbf{q}=2 \sum_{k=1}^{K} \mathbf{R}_{k}\left(\mathbf{R}_{k} \mathbf{q}-d_{k} \mathbf{t}\right)=0$ to yield a new value of $\mathbf{q}$ :

$$
\mathbf{q} \leftarrow \mathbf{H}\left(\sum_{k=1}^{K} d_{k} \mathbf{R}_{k}\right) \mathbf{t}
$$

where $\mathbf{H}=\left[\sum_{k=1}^{K} \mathbf{R}_{k}^{2}\right]^{-1}$ and $e \leftarrow f$ denotes replacing $e$ by $f$.

Stage 2: Freeze both $\mathbf{t}$ and $\mathbf{q}$ and adjust $\mathbf{d}$. Utilizing the property that $\|\mathbf{d}\|=1$ and considering the Lagrangian 
function

$$
J_{\lambda_{d}}=J+\lambda_{d}\left(\sum_{k=1}^{K} d_{k}^{2}-1\right)
$$

where $\lambda_{d}$ is the Lagrange multiplier, to obtain a new value of $\mathbf{d}$

$$
\mathbf{d} \leftarrow \frac{\mathbf{u}}{\|\mathbf{u}\|}, \quad \mathbf{u}=\left[\mathbf{r}_{1}^{T} \mathbf{t}, \mathbf{r}_{2}^{T} \mathbf{t}, \ldots, \mathbf{r}_{K}^{T} \mathbf{t}\right]^{T}
$$

where $\mathbf{r}_{k}=\mathbf{R}_{k} \mathbf{q}$.

Stage 3: Freeze both $\mathbf{q}$ and $\mathbf{d}$ and adjust $\mathbf{t}$. Using $\|\mathbf{t}\|=1$ and exploiting the Lagrangian function

$$
J_{\lambda_{t}}=J+\lambda_{t}\left(\mathbf{t}^{T} \mathbf{t}-1\right)
$$

to obtain the adjustment for $\mathbf{t}$

$$
\mathbf{t} \leftarrow \frac{\mathbf{v}}{\|\mathbf{v}\|}, \quad \mathbf{v}=\sum_{k=1}^{K} d_{k} \mathbf{r}_{k}
$$

These three stages are repeated until the cost function (5) converges, and one source can be extracted according to (4). For the later presented results on ECG signals, five iterations are typically sufficient and no problem with ill-convergence has been experienced. This, however, depends on the dimensions of the subspace that is being extracted [18]. After extracting one source, a deflation procedure is employed to remove it from the mixture as follows [15]:

$$
\mathbf{x}_{i+1}(t)=\mathbf{Z}^{T} \mathbf{x}_{i}(t), \quad \mathbf{x}_{1}(t)=\mathbf{x}(t)
$$

where $\mathbf{x}(t)$ is the original observation signal defined in (1), and

$$
\mathbf{Z}=\mathbf{I}-\frac{\mathbf{R}_{0(i)} \mathbf{w} \mathbf{w}^{T}}{\sigma_{y}^{2}}
$$

where $\mathbf{R}_{0(i)}=E\left[\mathbf{x}_{i}(t) \mathbf{x}_{i}^{T}(t)\right], \mathbf{I}$ is the $N \times N$ identity matrix, and $\sigma_{y}^{2}=E\left[y^{2}\right]$.

The autocorrelation matrix is then updated as

$$
\mathbf{R}_{0(i+1)}=\mathbf{Z}^{T} \mathbf{R}_{0(i)} \mathbf{Z}
$$

before another source can be extracted following the same procedure, using (6)-(13). An alternative way to obtain a deflation matrix is to design a matrix $\mathbf{Z}=\left[\mathbf{z}_{1}, \mathbf{z}_{2}, \ldots, \mathbf{z}_{N-1}\right]$ whose columns $\mathbf{z}_{i}$ span the subspace orthogonal to the estimated source direction $\mathbf{t}$, i.e., $\mathbf{z}_{i} \perp \mathbf{t}$ for $1 \leq i \leq N-1$. This latter approach can speed up the algorithm in the case of slow convergence.

This extraction algorithm is computationally simple when compared with one stage of other algorithms, such as those proposed in [16] that extract the sources one by one using fourthorder cumulants. It is, however, worth noting that the iterative extraction algorithm for estimating one source at a time in our study, in fact, replaces the joint diagonalization procedure in the SOBI algorithm [9], whereby the computation is simplified since full eigen-decomposition is not required. Nonetheless, performing the iterative procedure in our method is very similar to the procedure that is carried out within techniques that calculate the first (or the first few) eigenvalues [21]. In the next section, we extend this algorithm to the extraction of periodic signals with time-varying period.

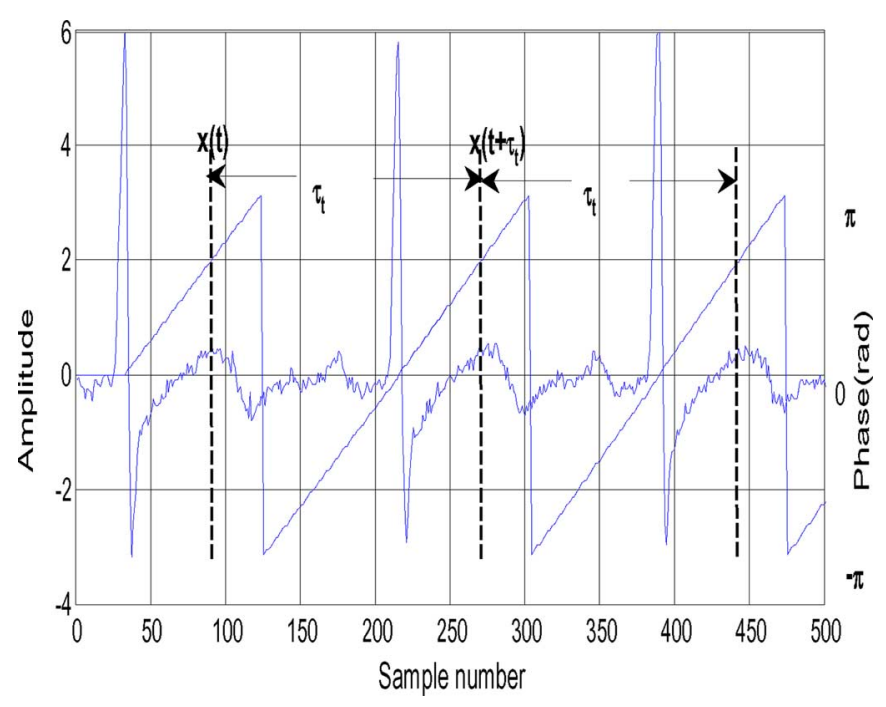

Fig. 1. Demonstration of phase allocation procedure first proposed in [20] for computing $\tau_{t}$. The sawtooth signal depicts the phase signature $\theta(t)$ ranging from $-\pi$ to $\pi$. The peaks positions are assigned to $\theta(t)=0$. For each period of the signal, half of the signal samples are assigned to $\theta(t)$, ranging from $-\pi$ to 0 , and the other half is assigned to $\theta(t)$, ranging from 0 to $\pi$. Typically, a sample at time instant $t$ is compared with the sample at $t+\tau_{t} . \tau_{t}$ is recalculated on a cycle-by-cycle basis.

\section{SEquential EXTRACTION Algorithm FOR QuAsi-Periodic Signals With TIME-VARYing PERIOD}

Successful minimization of the cost function (5) in concert with (4) leads to the extraction of any one source. It is not possible to extract the source of interest (SoI) unless some additional information is known a priori. The SoI in our case is a quasiperiodic signal of varying period duration. If the fundamental period, or its approximation, of the SoI is fixed and known, then the algorithm can be made to focus only on this specific source. This is based on the fact that if the fundamental period is, say, $\tau$ samples, then its autocorrelation matrix will have the same value at time lags corresponding to integer multiples of $\tau$. Hence, the autocorrelation matrices $\mathbf{R}_{k} \mathrm{~s}$, as computed in (2), can jointly be diagonalized at time lags $\tau, \ldots, K \tau$ along with the constraint $d_{1}=d_{2}=\cdots=d_{K}$.

However, if the SoI has a period that varies from period to period (see, for instance, Fig. 5), then to jointly diagonalize the $\mathbf{R}_{k}$ s at time lags $\tau, \ldots, K \tau$ and applying the extraction algorithm would invariably result in erroneous results. Therefore, a method has to be developed that effectively matches the variations in the period of the SoI.

\section{A. Proposed Method}

The method, recently proposed in [20] for multichannel ECG decomposition, entails detecting the peaks of the quasi-periodic signal that are assumed to define the period of the SoI, as is the case in ECG signals, and allowing a linear phase signature $\theta(t)$, to span the range from $-\pi$ to $\pi$, between the peaks. The phase signature is then allocated to each sample of the signal, with the positions of the R-peaks being fixed at $\theta(t)=0$, as shown in Fig. 1. It follows that the samples corresponding to a certain specific phase angle are compared along the signal. For 


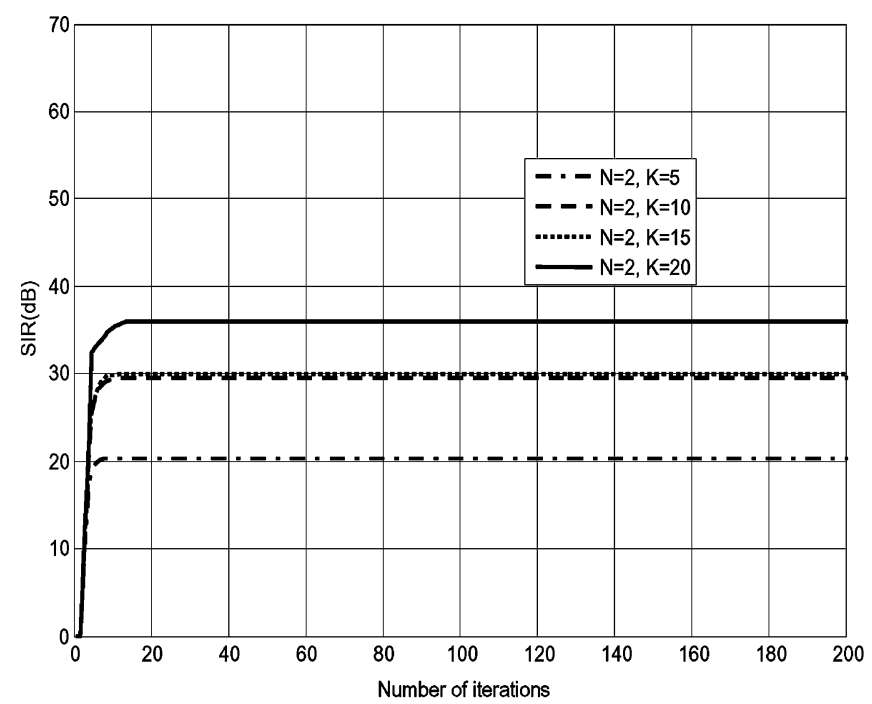

(a)

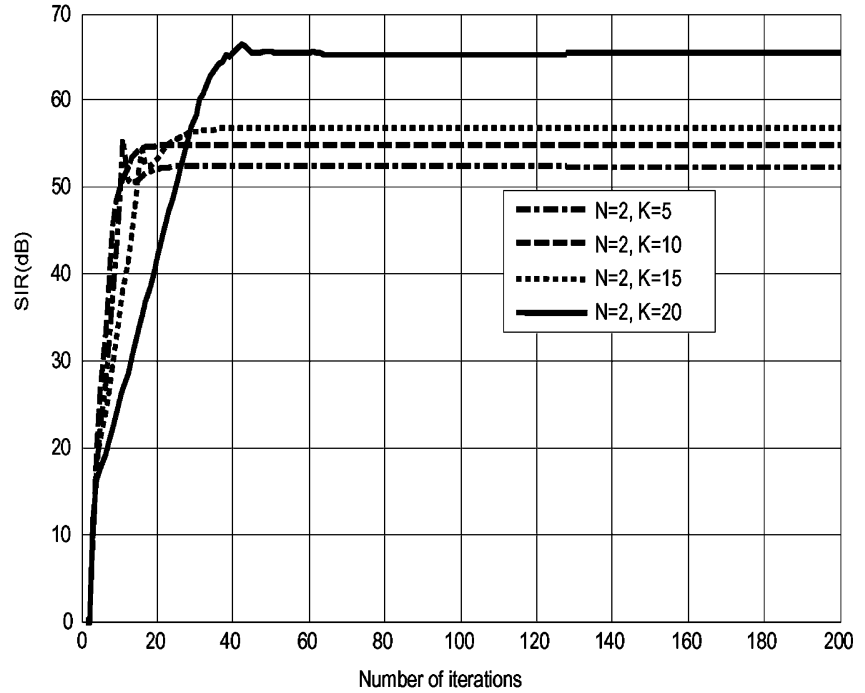

(b)

Fig. 2. SIR $(\mathrm{dB})$ versus the number of iterations for both fixed and time-varying extraction algorithms for the case of noise-free BSE, averaged over 250 independent runs when extracting the first source signal. $N$ represents the number of signals while $K$ represents the number of autocorrelation matrices used. SIR performance improves as the number of matrices increases. The SIR performance of the time-varying period algorithm almost doubles that of the fixed period algorithm. (a) SIR(dB) for extraction algorithm using fixed period algorithm. (b) SIR(dB) for extraction algorithm using time-varying period, notice the range on the SIR axis.

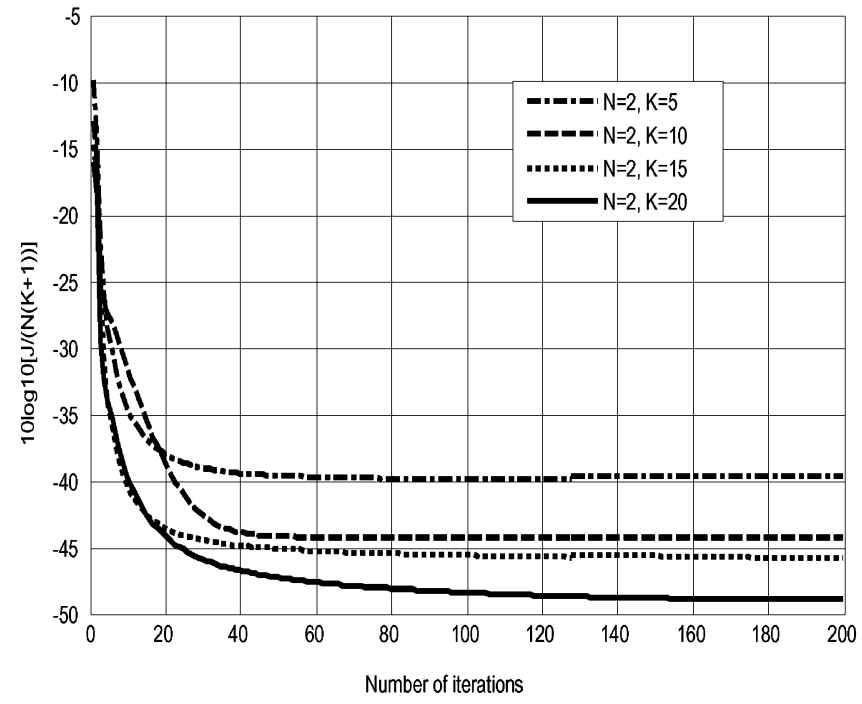

(a)

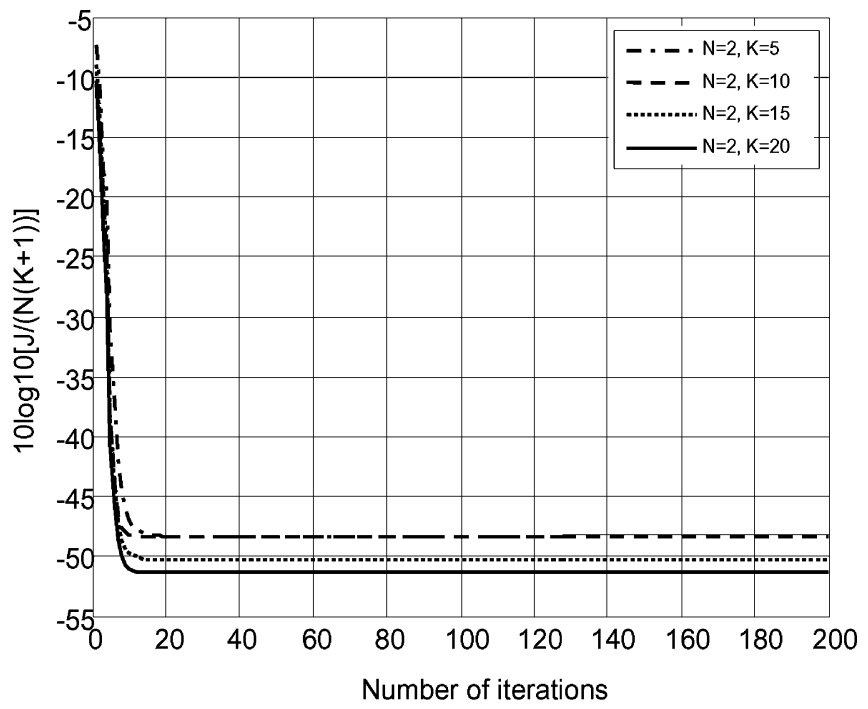

(b)

Fig. 3. $J(\mathbf{t}, \mathbf{q}, \mathbf{d}) / N(K+1)(\mathrm{dB})$ versus number of iterations for both fixed and time-varying extraction algorithms for the case of noise-free BSE, averaged over 250 independent runs when extracting the first source signal. $N$ represents the number of signals while $K$ represents the number of autocorrelation matrices used. The proposed algorithm converges faster than the fixed-period algorithm. (a) $J(\mathbf{t}, \mathbf{q}, \mathbf{d}) / N(K+1)(\mathrm{dB})$ for extraction algorithm using the fixed period algorithm. (b) $J(\mathbf{t}, \mathbf{q}, \mathbf{d}) / N(K+1)(\mathrm{dB})$ for extraction algorithm using the time-varying period algorithm.

example, in Fig. 1, for the phase angle of $2 \mathrm{rad}$, the samples at time instant $t$ and $t+\tau_{t}$ are compared accordingly. Therefore, in the sequential algorithm explained in Section II, we can redefine the following key equations.

1) The autocorrelation matrix in (2)

$$
\tilde{\mathbf{R}}_{\tau_{t}}=E_{t}\left[\mathbf{x}(t) \mathbf{x}^{T}\left(t-\tau_{t}\right)\right]
$$

where $E_{t}[\cdot]$ denotes averaging over $t$, and

$$
\tau_{t}=\min \{\tau \mid \theta(t+\tau)=\theta(t), \tau>0\} .
$$

2) The cost function in (5) is again exploited

$$
[\hat{\mathbf{t}}, \hat{\mathbf{q}}, \hat{\mathbf{d}}]=\arg \min _{\mathbf{t}, \mathbf{q}, \mathbf{d}} J(\mathbf{t}, \mathbf{q}, \mathbf{d})
$$

where $J(\mathbf{t}, \mathbf{q}, \mathbf{d})=\sum_{p=1}^{K}\left\|\tilde{\mathbf{R}}_{p \tau_{t}} \mathbf{q}-d_{p} \mathbf{t}\right\|^{2}$, where the $\tilde{\mathbf{R}}_{p \tau_{t}}$ terms are also calculated as time averages.

Therefore, the autocorrelation matrix and the cost function now take into account the variable period $\tau_{t}$, which is calculated from $\theta(t)$ from cycle-to-cycle of the signal. This leads to a new algorithm for extracting SoI with a variable period duration. 


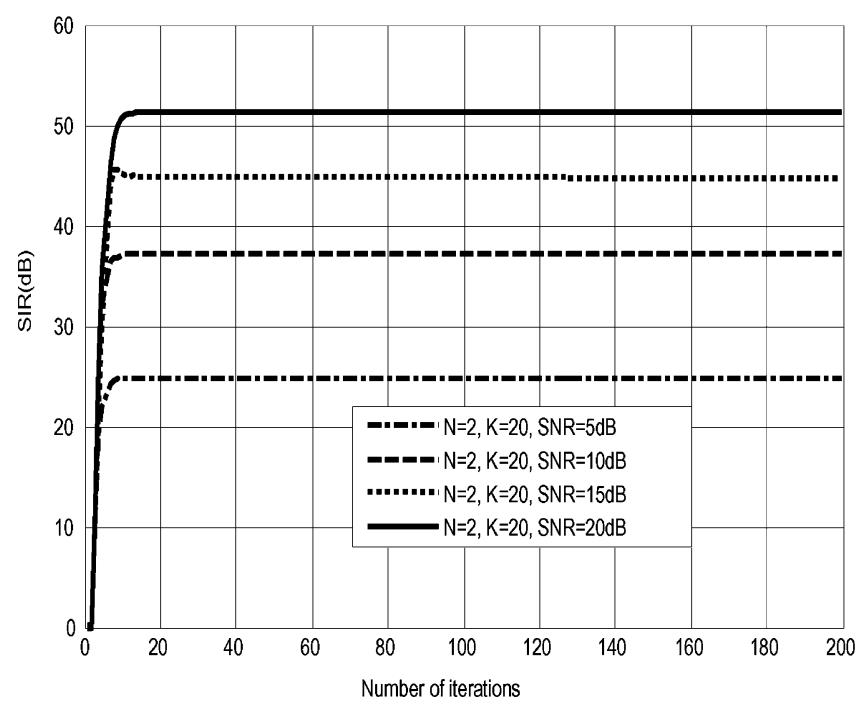

(a)

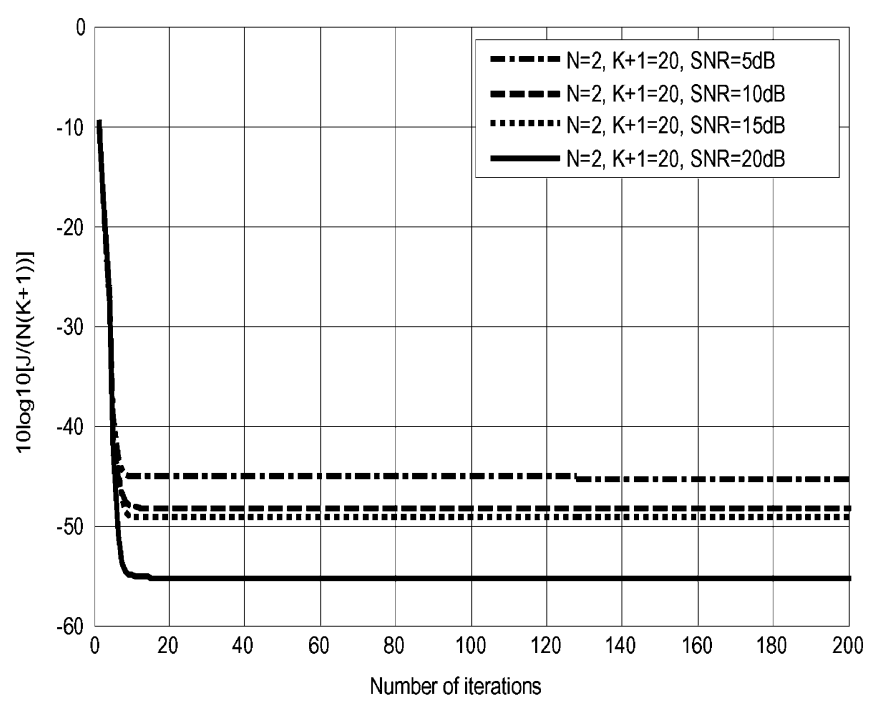

(b)

Fig. 4. $J(\mathbf{t}, \mathbf{q}, \mathbf{d}) / N(K+1)(\mathrm{dB})$ and $\mathrm{SIR}(\mathrm{dB})$ versus number of iterations using time-varying extraction algorithm for the case of noisy BSE, averaged over 250 independent runs when extracting the first source signal. $N$ represents the number of signals while $K$ represents the number of autocorrelation matrices used. (a) SIR(dB) for the extraction algorithm using time-varying period algorithm for different SNRs on observations. Note the degradation in SIR(dB) performance as a function of SNR(dB). (b) $J(\mathbf{t}, \mathbf{q}, \mathbf{d}) / N(K+1)(\mathrm{dB})$ for the extraction algorithm using the time-varying period algorithm for different SNRs on observations, note that the degradation in convergence performance as a function of reduction in $\operatorname{SNR}(\mathrm{dB})$.

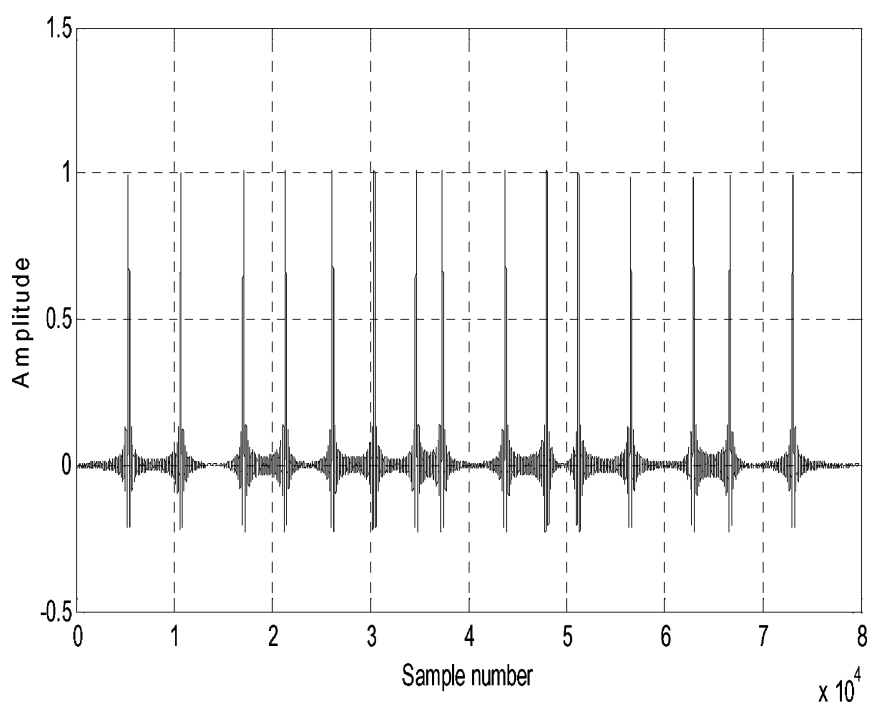

Fig. 5. Synthetic periodic signal designed to have considerable period variations. This signal acts as an SoI after mixing it with WGN.

The main difference in the algorithm of Section II and the one proposed in this paper is the way in which the time-lagged autocorrelation matrix $\tilde{\mathbf{R}}$ is computed, which, in turn, leads to the redefinition of the cost function (5).

In this algorithm, the autocorrelation matrices are calculated at varying time lags $\tau_{t}$ rather than at fixed time lags. Thus, after performing peak detection, and calculating the $\theta(t)$ and the time-varying $\tau_{t}$, each autocorrelation matrix is calculated by computing correlations between sample points $t$ and their dual samples $t+\tau_{t}$ across the entire signal length, and then, averaging over the number of correlation and phase angle points. The resulting $\tilde{\mathbf{R}}$ s are used in the sequential algorithm of Section II to extract the SoI from multichannel mixtures.

\section{Simulation Results}

Computer simulations were carried out to illustrate the performance of the proposed method, and were compared to the one proposed recently in [17], which is based on a fixed period of the SoI.

\section{A. Signal-to-Interference Ratio and the Cost Function}

The performance of the algorithm was evaluated by the following.

1) The peak signal-to-interference ratio (SIR) in decibels is given by

$$
\mathrm{SIR}(\mathrm{dB})=10 \log _{10} \frac{\max \left(\left|v_{i}\right|^{2}\right)}{\sum_{i=1}^{N}\left|v_{i}\right|^{2}-\max \left(\left|v_{i}\right|^{2}\right)}
$$

where $\left[v_{1}, v_{2}, \ldots, v_{N}\right]=\mathbf{q}^{T} \mathbf{A}$ is the global transform vector, and (17) is evaluated by first calculating the average of SIR in a linear scale, and then, converting to decibels. For completeness, we note that from (1) and (4)

$$
y(t)=\mathbf{q}^{T} \mathbf{A} \mathbf{s}(t)=v_{1} s_{1}(t)+v_{2} s_{2}(t)+\cdots+v_{N} s_{N}(t)
$$

2) The cost function in decibels given by $J(\mathbf{t}, \mathbf{q}, \mathbf{d}) / N(K+$ $1)$.

In the simulation, blind extraction of the ECG signal obtained from DaISY database (available at: http://homes.esat.kuleuven.be/smc/daisy/) was considered. The 2500-sample-long clean ECG signal, sampled at $500 \mathrm{~Hz}$, was concatenated to form a 7500-sample-long signal. It is worth noting that no discontinuity problems were experienced when 


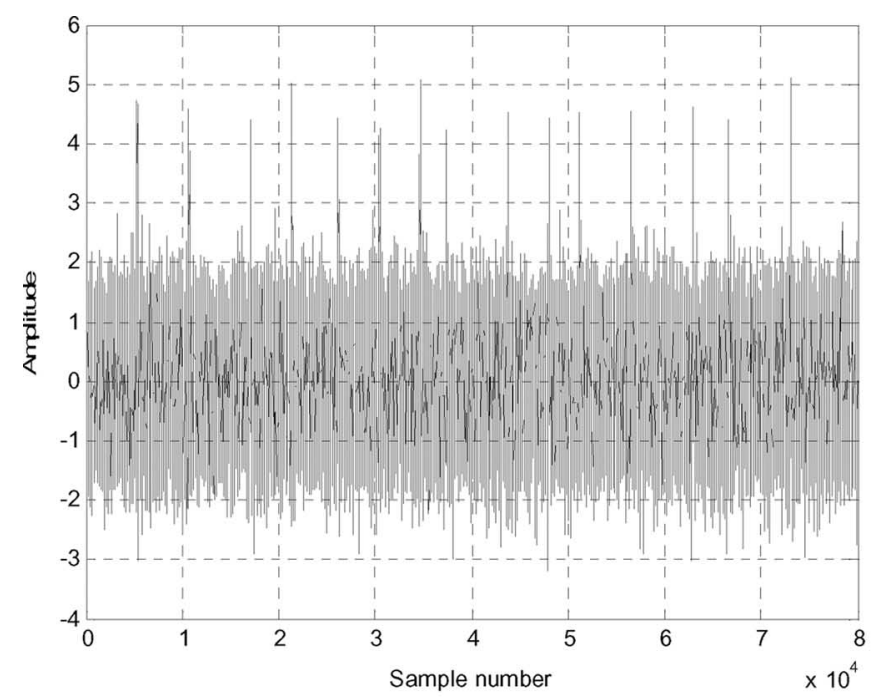

(a)

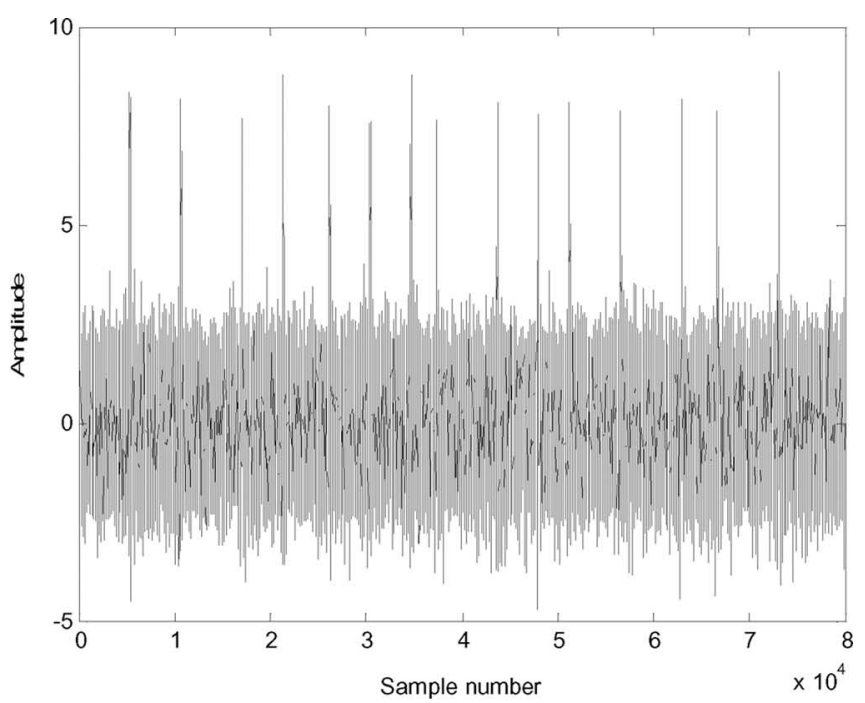

(b)

Fig. 6. Mixtures of synthetic periodic signal with time-varying period and WGN, generated by a mixing matrix A with elements drawn from a standardized Gaussian distribution. The synthetic periodic signal is designed to have significant period variations. The aim is to extract the synthetic periodic signal (Fig. 5). (a) Mixture 1 of synthetic signal with time-varying period and WGN. (b) Mixture 2 of synthetic signal with time-varying period and WGN.

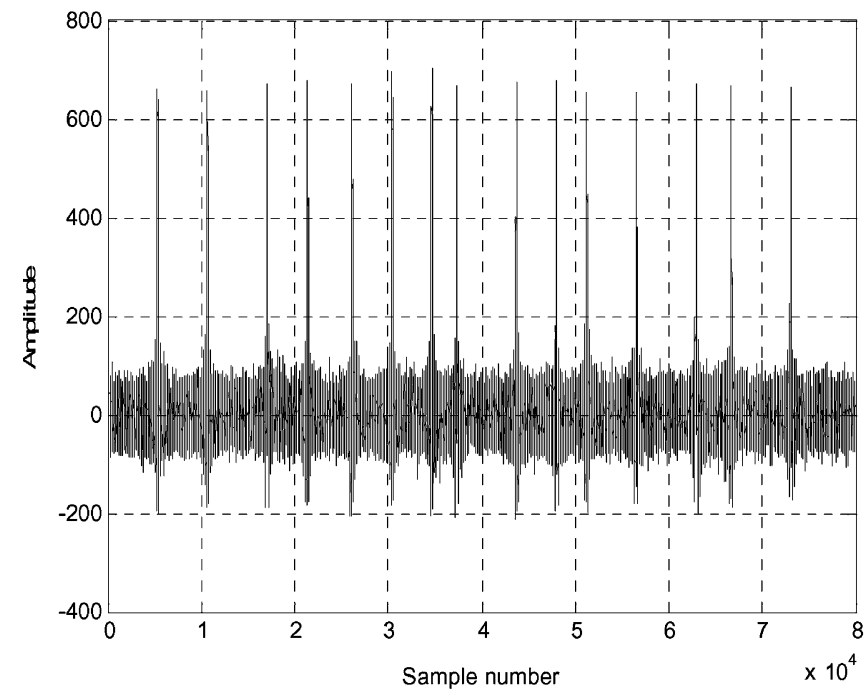

(a)

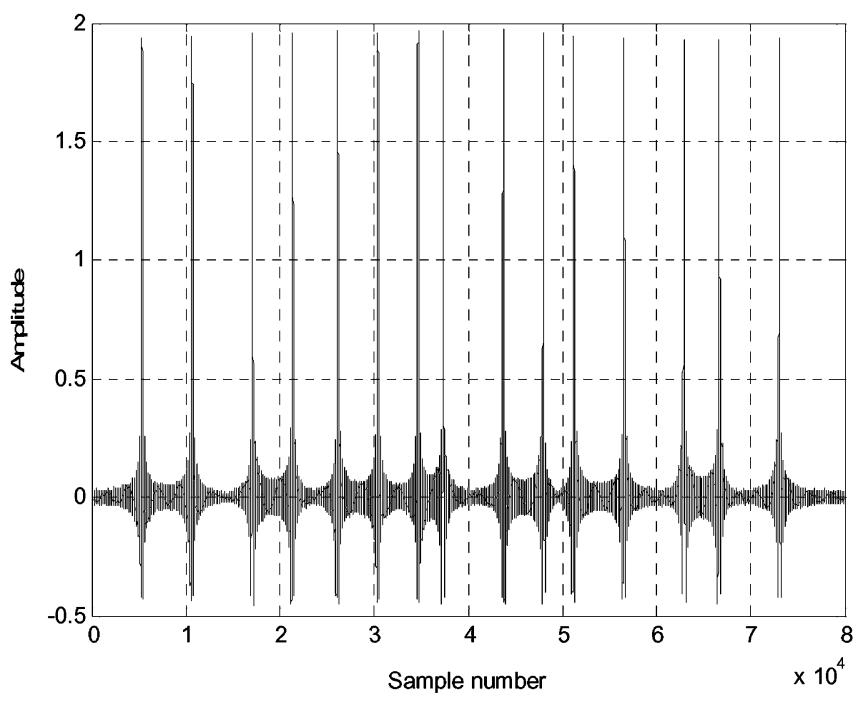

(b)

Fig. 7. Extracted synthetic signals using algorithms with the fixed and time-varying period. Clearly, the algorithm employing time-varying period much better reconstructs the synthetic signal and we can see the variations in the signal period. The algorithm using the fixed period locks onto the noise component and results in a poorly reconstructed signal. (a) Extracted synthetic signal using the algorithm with fixed period. (b) Extracted signal using the algorithm with time-varying period.

concatenating the signal. The ECG signal was mixed with white Gaussian noise (WGN) by a mixing matrix $\mathbf{A}$ with elements drawn from a standardized Gaussian distribution. Fig. 2(a) and (b) shows the $\operatorname{SIR}(\mathrm{dB})$ versus the number of iterations averaged over 250 independent runs when extracting the SoI, assuming a fixed and time-varying period, respectively. Fig. 3(a) and (b) represents the corresponding cost function performance in decibels for both cases. $N$ and $K$, shown in the figures, represent the number of original signals and the number of autocorrelation matrices used, respectively. Thus, the performance criteria were evaluated for $N=2$ and $K$ set to $5,10,15$, and 20 accordingly.
It is seen from Fig. 3(a) and (b) that the proposed algorithm converges faster than the fixed-period algorithm, with convergence improving with the number of matrices used. The SIR performance also improves as the number of matrices is increased. As seen from Fig. 2(b), there is a marked increase in the SIR performance for the proposed algorithm. In fact, the SIR performance of the proposed algorithm almost doubles that of the algorithm using a fixed period. For instance, from Fig. 2(a) and (b), the maximum SIR when assuming fixed and time-varying period, and using 20 matrices is 33 and $65 \mathrm{~dB}$, respectively. This underlines the motivation for our study, since by exploiting the 


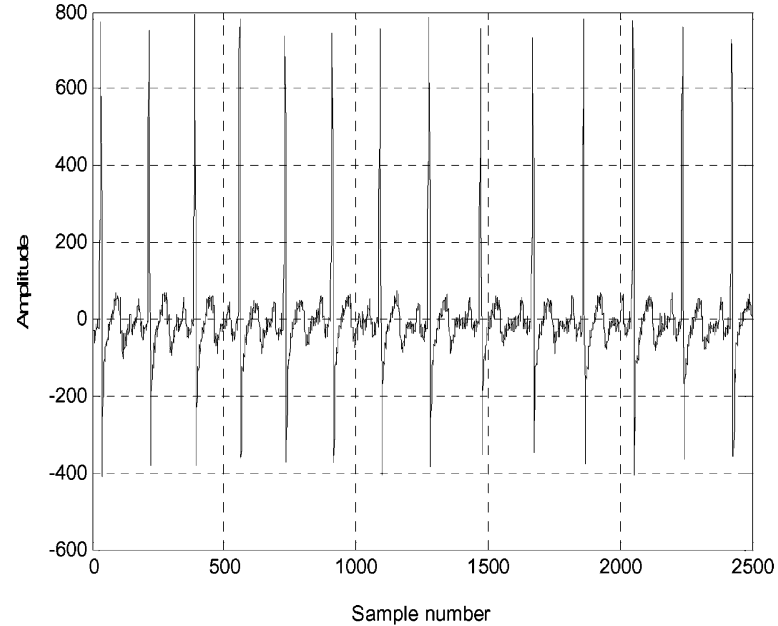

(a)

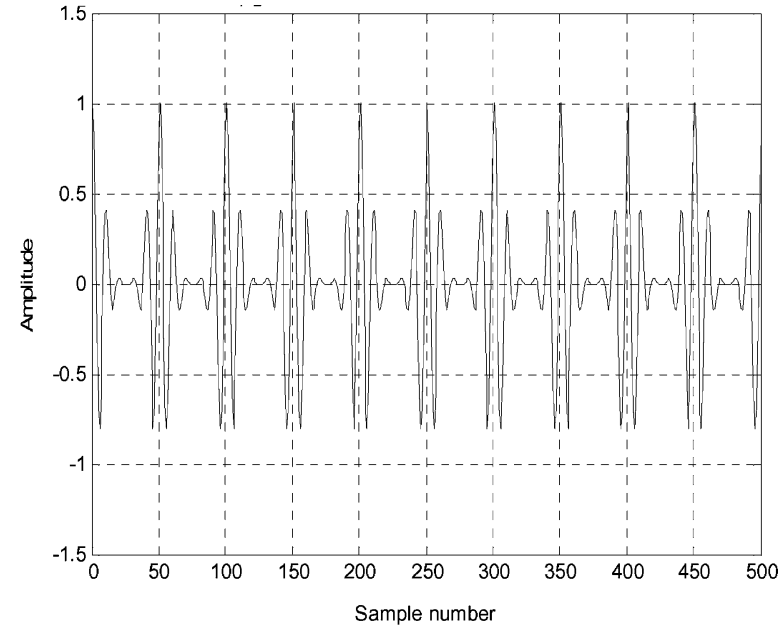

(b)

Fig. 8. ECG and a zoomed-in portion of a synthetic pure periodic signal whose repetition frequency is not a multiple of that of the ECG. These signals are combined by a mixing matrix $\mathbf{A}$ with elements drawn from standardized Gaussian distribution. The aim was to extract the ECG signal that has a time-variant period. (a) ECG signal that is to be extracted after mixing it with a synthetic pure periodic signal. The signal has slight variations in period durations. (b) Synthetic pure periodic signal that is mixed with the ECG signal. It is designed to be nonharmonically related to the ECG signal.

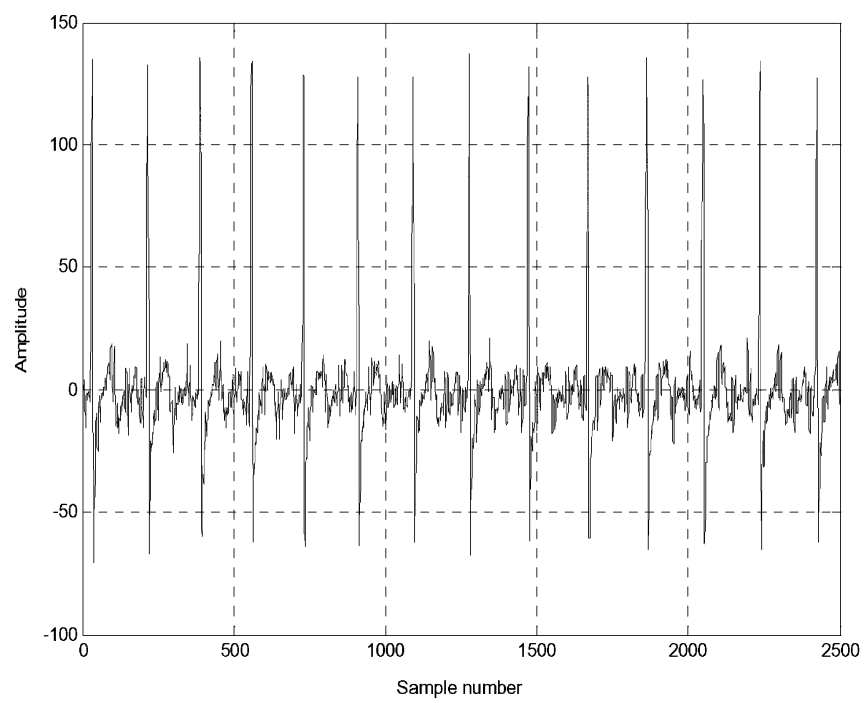

(a)

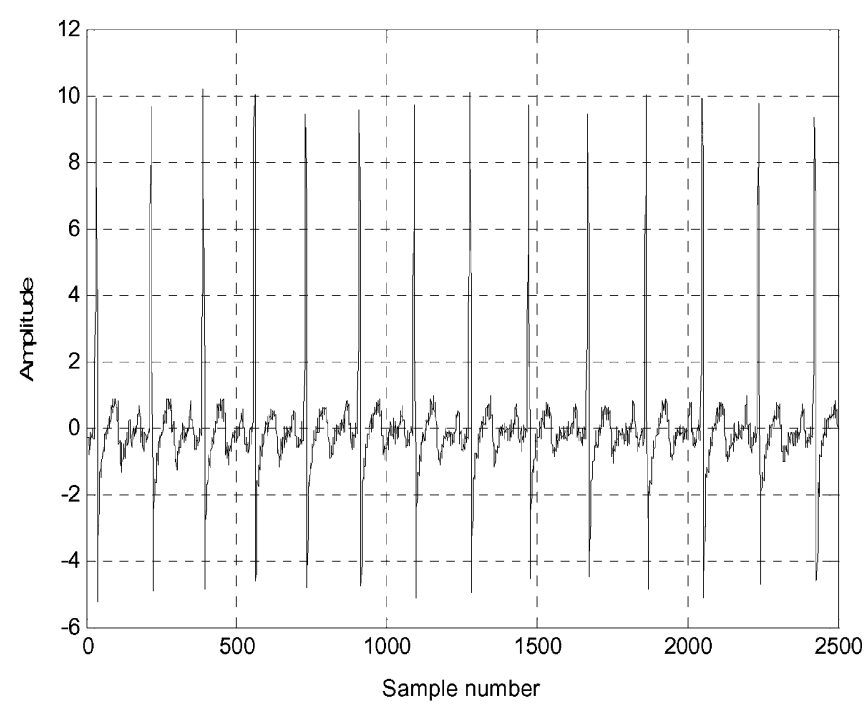

(b)

Fig. 9. Extracted ECG signals using algorithms with fixed and time-varying period. The algorithm employing time-varying period reconstructs the ECG signal

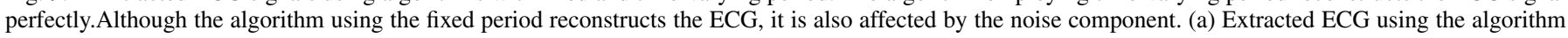
employing fixed period. (b) Extracted ECG using the algorithm employing time-varying period.

nonstationarity of the source, captured in the varying period, we are achieving improved SIR performance for the same fast convergence performance.

The performance of our algorithm was also investigated using different SNRs on mixture signals for the case of the noisy model given by (1). Fig. 4(a) and (b) shows SIR(dB) and convergence performance as a function of $\operatorname{SNR}(\mathrm{dB})$, respectively. It is seen from the figures that the performance degrades as more independent noise is added to the mixtures, i.e., as SNR(dB) reduces. It is however seen from Figs. 2(a) and 4(a) that our algorithm, when applied to the noisy BSE, still outperforms (at least at SNR of $10 \mathrm{~dB}$ ) the one using a fixed period in terms of $\operatorname{SIR}(d B)$, when applied to noise-free BSE.

\section{B. Extraction of Synthetic Variable Period Signal}

This simulation considers the extraction of a synthetic, deterministic signal, with time-varying period (Fig. 5). The signal is mixed with WGN in the same manner as before. Both algorithms are run to extract the periodic signal. Fig. 6(a) and (b) shows the mixtures while Fig. 7(a) and (b) shows the extracted periodic signal using algorithms employing the fixed and the 
time-varying periods, respectively. As seen from the latter figures, when a fixed period is used in the algorithm, the algorithm not only recovers the signal, but also heavily locks onto the noise component, by running the proposed algorithm, which incorporates the time-varying period, accurate reconstruction is achieved, as confirmed by Fig. 7(b).

\section{Separation of Two Periodic Signals}

Another investigation was performed considering the separation of two nonharmonically related periodic signals, i.e., the ECG signal having varying period duration [see Fig. 8(a)], mixed with a synthetic purely periodic signal shown in Fig. 8(b). The SoI in this case is the ECG signal. The recovered ECG signals are shown in Fig. 9(a) and (b) for algorithms employing fixed and time-varying periods, respectively. As seen in Fig. 9(b), the proposed algorithm recovers the ECG completely. This shows that the algorithm works, not only for a periodic signal contaminated with WGN, but also for separating periodic signals. This can be likened to biomedical applications, such as the extraction of the HSS from lung sound recordings, where both the HSS and lung sound signal (LSS) have distinct periodicity but are generally not harmonically related. Another example is the extraction of fetal ECG signals from maternal abdominal sensors that are highly contaminated with the maternal ECG [20].

\section{Application of the Proposed Algorithm to SEPARATION OF THE HEART BEAT SOUND SignAL FROM REAL LUNG SOUND RECORDINGS}

In this section, we demonstrate the applicability of the proposed algorithm to the extraction of the HSS from real recorded lung sound recordings. The dataset comprises two synchronized recordings obtained from channel (1), front left chest (heart location), and channel (2), front right chest, by digital stethoscopes sampled at $44100 \mathrm{~Hz}$ with 16-bit resolution. It is worth noting that, in order to use the algorithm, a clean reference signal with clear distinct peaks is required such that the peaks could automatically be detected using the readily available peak detection algorithm. The clean reference signal in our case would be the ECG signal that is synchronized with the two channel recordings. However, since this ECG was not available, we reverted to using "manual" peak detection where data from channel (1) was prefiltered prior to using our judgement about the occurrence of the peaks in the data. Using the resulting peak locations, we calculated both the $\theta(t)$ and the $\tau_{t}$, which are necessary to compute the $\tilde{\mathbf{R}}$ s for two channel data. The algorithm was run with the two raw recordings as mixture signals. The two recordings are shown in Fig. 10(a) and (b). The recovered HSSs for when both the fixed and time-varying algorithms are used are shown in Fig. 10(c) and (d), respectively. The HSS recovered from using the time-varying algorithm has clear distinct peaks depicting a better estimate of the actual HSS. Using the fixed-period algorithm results in a noisy reconstructed HSS. These results have been further corroborated by listening tests. In the listening tests, five subjects of normal hearing ability were asked to listen to both the recovered HSSs and to comment on their intelligibil-
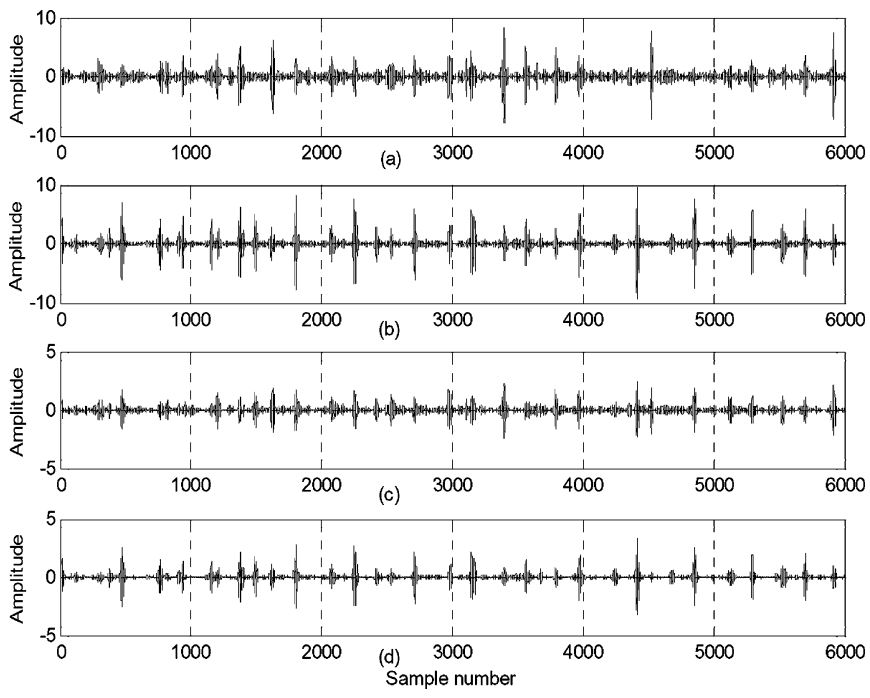

Fig. 10. Extraction of HSS from lung sound recordings. (a) and (b) Lung sound recordings (also called mixtures since each contain both heart and lung sound signals). The aim is to extract the HSS from the recordings. (c) and (d) Resulting extracted HSS for algorithms employing fixed and time-varying period, respectively, the definition of the signal in (d) is much improved.

ity. All subjects observed that although it was evident that the recovered signals were HSSs, the one recovered when using the fixed period was less intelligible due to the presence of noise.

\section{SUMmARY AND CONCLUSION}

The performance of the BSE algorithm depends on a priori knowledge of the source signal. Knowledge of the period of the SoI helps to extract the source signal of interest from the mixtures. In this paper, a novel sequential algorithm using secondorder statistics for the BSE of quasi-periodic source signals, which exploits the temporal, time-varying, quasi-periodicity of the source signals, was introduced. The algorithm was based on partial approximate joint diagonalization of autocorrelation matrices at time-varying lag $\tau_{t}$, which is recalculated on a cycle-bycycle basis. The algorithm is suitable for multichannel decomposition of periodic signals with or without a time-varying period. Simulation results suggest that if the SoI has a time-varying period, then using an algorithm employing a fixed period results in erroneous results. Results from other investigations show that the algorithm is suitable for removing a HSS from lung sound recordings where the periodic variation in the heart beat has been extracted manually. However, with the availability of a suitably clean ECG signal, which would be synchronous with the underlying heart sound within the phonocardiogram signals, significant improvements might be possible and the heart beat period extraction could then be automated. Furthermore, due to the multidimensional nature of the ECG, the results for multichannel recordings may be improved by using more ECG reference signals [19] which could thereby better exploit the subcomponents of the ECG recording, i.e., the P, QRS, and the T-waves.

The cost function in (5), proposed in [13], has some limitations. First, its convergence is rather difficult to prove analytically in the time domain. Second, there are some questions regarding its exact formulation and constraints imposed on the 
associated vector norms. This, together with increasing the number of channels for lung sound recordings and exploring other algorithms based on cost functions that do not exhibit the aforementioned shortcomings, forms part of our future work. In conclusion, this paper is nonetheless a step forward in overcoming the time-varying periodic characteristic of many nonstationary biomedical measurements such as the HSSs, thereby allowing separation using information about a signal's periodicity.

\section{REFERENCES}

[1] C. Jutten and J. Hérault, "Blind separation of sources, Part I: An adaptive algorithm based on a neuromimetic architecture," Signal Process., vol. 24, pp. 1-10, 1991.

[2] J. F. Cardoso and A. Souloumiac, "Blind beamforming for non-Gaussian signals," in Proc. Inst. Elect. Eng., vol. 140, pp. 3362-3370, 1993.

[3] P. Comon, "Independent component analysis, a new concept?," Signal Process., vol. 36, pp. 287-314, 1994.

[4] S. Amari, A. Cichocki, and H. H. Yang, "A new learning algorithm for blind signal separation," Adv. Neural Inf. Process. Syst., vol. 8, pp. 752763, 1996.

[5] L. Parra and C. Spence, "Convolutive blind source separation of nonstationary sources," IEEE Trans. Speech Audio Process., vol. 8, no. 3, pp. 320-327, May 2000.

[6] K. Matsuoka, M. Oya, and M. Kawamoto, "A neural net for blind separation of nonstationary signals," Neural Netw., vol. 8, pp. 411-419, 1995.

[7] D. T. Pham and J. F. Cardoso, "Blind separation of instantaneous mixtures of non stationary sources," IEEE Trans. Signal Process., vol. 49, no. 9, pp. 1837-1848, Sep. 2001.

[8] L. Molgedey and H. G. Schuster, "Separation of a mixture of independent signals using time delayed correlations," Phys. Rev. Lett., vol. 72, pp. 3634-3637, 1994.

[9] A. Belouchrani, K. Abed-Meraim, J. F. Cardoso, and E. Moulines, "A blind source separation technique using second-order statistics," IEEE Trans. Signal Process., vol. 45, no. 2, pp. 434-444, Feb. 1997.

[10] S. Choi and A. Cichocki, "Blind separation of nonstationary sources in noisy mixtures," Electron. Lett., vol. 36, pp. 848-849, 2000.

[11] A. Belouchrani and A. Cichocki, "A robust procedure in blind source separation context," Electron. Lett., vol. 36, pp. 2050-2051, 2000.

[12] M. G. Jafari, W. Wang, J. A. Chambers, T. Hoya, and A. Cichocki, "Sequential blind source separation based exclusively on second-order statistics developed for a class of periodic signals," IEEE Trans. Signal Process., vol. 54, no. 3, pp. 1028-1040, Mar. 2006.

[13] X. Li and X. Zhang, "Sequential blind extraction adopting second-order statistics," IEEE Signal Process. Lett., vol. 14, no. 1, pp. 58-60, Jan. 2007.

[14] R. T. Behrens and L. L. Scharf, "Signal processing applications of oblique projection operators," IEEE Trans. Signal Process., vol. 42, no. 6, pp. 1413-1424, Jun. 1994.

[15] A. Cichocki and S. Amari, Adaptive Blind Signal and Image Processing: Learning Algorithms and Applications. New York: Wiley, 2002, pp. 191-192.

[16] N. Delfosse and P. Loubaton, "Adaptive blind separation of independent sources: A deflation approach," Signal Process., vol. 45, pp. 59-83, 1995.

[17] T. Tsalaile, S. M. Naqvi, K. Nazarpour, S. Sanei, and J. A. Chambers, "Blind source extraction of heart sound signals from lung sound recordings exploiting periodicity of the heart sound," in Proc. ICASSP'08, pp. 461464.

[18] R. Sameni, C. Jutten, and M. B. Shamsollahi, "What ICA provides for ECG processing: Application to noninvasive fetal ECG extraction," in Proc. Int. Symp. Signal Process. Inf. Technol. (ISSPIT'06), pp. 656-661.

[19] R. Sameni, G. D. Clifford, C. Jutten, M. B. Shamsollahi. (2007). Multichannel ECG and Noise Modeling: Application to Maternal and Fetal ECG Signals. EURASIP J. Adv. Signal Process. [Online]. pp. 43 407-43 421. Available: http://www.hindawi.com/GetArticle. aspx?doi=10.1155/2007/43407.

[20] R. Sameni, C. Jutten, and M. B. Shamsollahi, "Multichannel electrocardiagram decomposition using periodic component analysis," IEEE Trans. Biomed. Eng., vol. 55, no. 8, pp. 1935-1940, Jul. 2008.

[21] G. H. Golub and C. F. Van Loan, Matrix Computation, 3rd ed. Baltimore, MD: Johns Hopkins Series in the Mathematical Sciences, 1996.

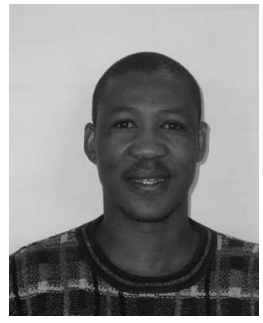

Thato Tsalaile (M'07) was born in Kanye, Botswana, in 1967. He received the B.Eng. degree in electrical and electronic engineering and the MBA degree in management from the University of Botswana, Gaborone, Botswana, in 1997 and 2004, respectively, the M.Sc. degree in electronic engineering from Cardiff University, Cardiff, U.K., in 2002, and the Diploma degree in project management from Damelin School of Management, Gaborone, in 1999. $\mathrm{He}$ is currently working toward the Ph.D. degree in statistical signal processing at Loughborough University, Loughborough, U.K.

From 1990 to 1994, he was an Electrical and Instrumentation Technician. From 1997 to 2000, he was a Project Engineer. In 2000, he joined the University of Botswana as a Lecturer, where, since 2000, he has been teaching digital signal processing and digital systems design, and coordinating the industrial attachments of students.

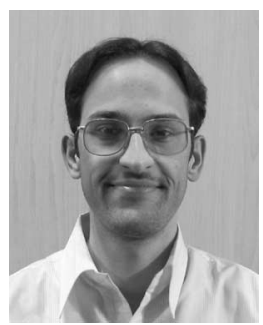

Reza Semeni (S'01) was born in Shiraz, Iran, in 1977. He received the B.Sc. degree in electronics engineering from Shiraz University, Shiraz, Iran, in 2000 , and the M.Sc. degree in bioelectrical engineering from Sharif University of Technology, Tehran, Iran, in 2003. He is currently working toward the $\mathrm{Ph} . \mathrm{D}$. degree in electrical engineering at Sharif University of Technology and the Institut National Polytechnique de Grenoble (INPG), Grenoble, France.

He has worked in industry on the design and implementation of digital electronics and software-defined radio systems. His current research interests include statistical signal processing and time-frequency analysis of biomedical recordings, modeling, filtering, and analysis of fetal cardiac signals.

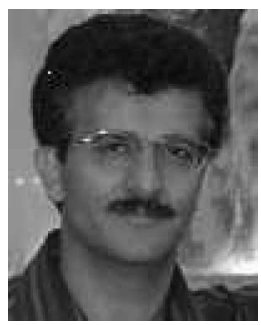

Saeid Sanei (M'97-SM'05) received the Ph.D. degree in biomedical signal and image processing from the Imperial College of Science, Technology, and Medicine, London, U.K., in 1991.

He has been a member of the academic staff in Iran, Singapore, and U.K. He is currently with the Center of Digital Signal Processing, Cardiff University, Cardiff, U.K. He is the author or coauthor of more than 170 papers in refereed journals and conference proceedings, and the author of the book Electroencephalography (EEG) Signal Processing. His current research interests include biomedical signal and image processing, adaptive and nonlinear signal processing, and pattern recognition and classification.

Dr. Sanei has been an Editor, a member of technical committees, and a reviewer for a number of journals and conference proceedings.

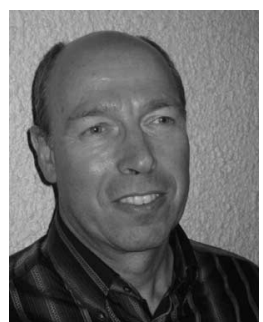

Christian Jutten (M'03-SM'06) received the Ph.D. and the Dr. Sci. degrees in electrical engineering from the Institut National Polytechnique of Grenoble, Grenoble, France, in 1981 and 1987, respectively.

From 1982 to 1989, he was an Associate Professor in the Electrical Engineering Department. During 1989, he was a Visiting Professor at the Swiss Federal Polytechnic Institute, Lausanne, Switzerland. He was a Full Professor in the Sciences and Technologies Department, Polytech'Grenoble, University Joseph Fourier of Grenoble, Grenoble. He is currently the Associate Director and the Head of the Department Images-Signal (DIS), Grenoble Images, Speech, Signal and Control Laboratory (GIPSA, 300 people), 
Grenoble, France. His current research interests include blind source separation, independent component analysis, and learning in neural networks, including theoretical aspects (separability, source separation in nonlinear mixtures), applications in signal processing (biomedical, seismic, speech), and data analysis. $\mathrm{He}$ is the author or coauthor of more than 45 papers published in international journals, three books, 16 invited papers, and 140 communications in international conferences.

Dr. Jutten was an Associate Editor of the IEEE TRANSACTIONS ON CIRCUITS AND SYSTEMS (CAS) from 1992 to 1994 and a Co-Organizer of the First International Conference on Blind Signal Separation and Independent Component Analysis,Aussois, France, January 1999. From 1996 to 1998, he was a Scientific Advisor for signal and images processing at the French Ministry of Research and for the French National Research Center from 2003 to 2006. He is a member of the technical committee "Blind Signal Processing" of the IEEE CAS society and the technical committee "Machine Learning for Signal Processing" of the IEEE Signal Processing society. He is a reviewer of main international journals (IEEE TRANSACTIONS ON SIGNAL PROCESSING, IEEE Signal Processing LETTERS, IEEE Transactions ON NEURAL NETWORKS, IEEE Transactions ON SignAl PROCESSING, IEEE TRANSACTIONS ON NEURAL COMPUTATION, IEEE TRANSACTIONS ON NEUROCOMPUTING, etc.) and conferences in signal processing and neural networks [the International Conference on Acoustics, Speech, and Signal Processing (ICASSP), the ISCASS, the European Signal Processing Conference (EUSIPCO), the International Joint Conference on Neural Networks (IJCNN), the Independent Component Analysis (ICA), the European Symposium on Artificial Neural Networks (ESANN), the International Work-Conference on Artificial Neural Networks (IWANN), etc.]. He was the recipient of the European Association for Signal Processing (EURASIP) Best Paper Award in 1992 and the Medal Blondel in 1997 from the French Electrical Engineering society (SEE) for his contributions in source separation and independent component analysis.

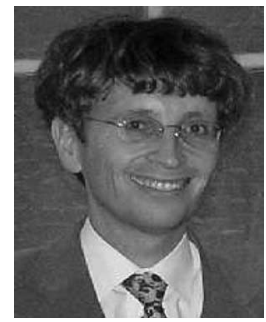

Jonathon Chambers (S'83-M'90-SM'98) was born in Peterborough, U.K., in 1960. He received the B.Sc. (Hons.) degree in electrical engineering from the Polytechnic of Central London, London, U.K., in 1985, and the Ph.D. degree in digital signal processing from the University of London, London, in 1990.

$\mathrm{He}$ was at Peterhouse, Cambridge University, U.K., and the Imperial College London, U.K. From 1979 to 1982, he was as an Artificer Apprentice in Action, Data, and Control in the Royal Navy. He has held academic and industrial positions at Bath University, Cardiff University, Imperial College London, King's College London, and Schlumberger Cambridge Research, U.K. In July 2007, he joined the Department of Electronic and Electrical Engineering, Loughborough University, Loughborough, U.K., as a Professor of Communications and Signal Processing, and currently leads the Advanced Signal Processing Research Group and a team of researchers in the analysis, design, and evaluation of novel algorithms for digital signal processing with application in acoustics, biomedicine, and wireless communications. He has authored or coauthored more than 300 research outputs including two monographs and more than 100 journal articles.

Dr. Chambers is a member of the IEEE Technical Committee on Signal Processing Theory and Methods and also the Technical Chair for the IEEE Workshop on Statistical Signal Processing 2009. He was the Chairman of the Institute for Electrical Engineers (IEE) Professional Group E5, Signal Processing. He was an Associate Editor for the IEEE SIGNAL PROCESSING LETTERS and is currently serving for a second term for the IEEE TRANSACTIONS ON SIGNAL PROCESSING 